\title{
Particle Collisions in the Lower Dimensional Rotating Black Hole Space-Time with the Cosmological Constant
}

\author{
Jie Yang, ${ }^{1}$ Yun-Liang Li, ${ }^{1}$ Yang Li, ${ }^{2}$ Shao-Wen Wei, ${ }^{1}$ and Yu-Xiao Liu' \\ ${ }^{1}$ Institute of Theoretical Physics, Lanzhou University, Lanzhou 730000, China \\ ${ }^{2}$ Department of Physics, Beijing Normal University, Beijing 100875, China \\ Correspondence should be addressed to Jie Yang; yangjiev@lzu.edu.cn
}

Received 3 January 2014; Accepted 14 February 2014; Published 17 March 2014

Academic Editor: Xiaoxiong Zeng

Copyright (c) 2014 Jie Yang et al. This is an open access article distributed under the Creative Commons Attribution License, which permits unrestricted use, distribution, and reproduction in any medium, provided the original work is properly cited. The publication of this article was funded by SCOAP ${ }^{3}$.

\begin{abstract}
We study the effect of ultrahigh energy collisions of two particles with different energies near the horizon of a $2+1$ dimensional BTZ black hole (BSW effect). We find that the particle with the critical angular momentum could exist inside the outer horizon of the BTZ black hole regardless of the particle energy. Therefore, for the nonextremal BTZ black hole, the BSW process is possible on the inner horizon with the fine tuning of parameters which are characterized by the motion of particle, while, for the extremal BTZ black hole, the particle with the critical angular momentum could only exist on the degenerated horizon, and the BSW process could also happen there.
\end{abstract}

\section{Introduction}

In the recent paper [1], Banãdos, Silk, and West proposed a mechanism (BSW process) that two particles may collide on the horizon of an extremal Kerr black hole with ultrahigh center-of-mass (CM) energy, although it was pointed out in $[2,3]$ that the collision in fact takes an infinite proper time. Moreover, there are astrophysical limitations preventing a Kerr black hole from being an extreme one, and the gravitational radiation and backreaction effects should also be included in this process. Due to the potential interest in exploring ultrahigh energy physics, the BSW process has been studied extensively in other kinds of black holes or naked singularities [4-29]. To achieve ultrahigh CM energy under the astrophysical limitation of maximal spin, the multiple scattering was taken into account in the nonextremal Kerr black hole $[7,15]$. Another more direct application is to consider different extreme rotating black holes, such as the Kerr-Newman black holes and the Sen black hole $[8,11]$. On the other hand, a general explanation of this BSW process was tried to give for a rotating black hole [19] and for other black holes [20,21]. Some efforts had also been made to draw some implications concerning the effects of gravity generated by colliding particles in [23].
However, all of the works mentioned above have been focused on the black holes embedded in the asymptotically flat space-time without cosmological constant. In our previous work [30], we had considered the BSW process in the background of the Kerr- (anti-) de Sitter black hole with nonzero cosmological constant and had found that the cosmological constant has an important effect on the result. For the case of the general Kerr-de Sitter black hole (with positive cosmological constant), the collision of two particles can take place on the outer horizon of the nonextremal black hole and the CM energy of collision can blow up arbitrarily if one of the colliding particles has the critical angular momentum. In the present paper, we extend the investigation of the BSW process to the background of a 2 +1 dimensional BTZ black hole [31], and our motivation is to examine whether the BSW effect remains valid in the lower dimensional case. Actually, in $[5,6]$, Lake had pointed out the divergence of the CM energy of particle collision on the inner horizon of the BTZ black hole, but the process was not discussed in detail. In this paper, we study this process in the BTZ black hole with circumstances.

This paper is organized as follows. In Section 2, we give a brief review of the BTZ black hole. In Section 3, we study the 
CM energy of the particle collision on the horizon and derive the critical angular momentum to blow up the CM energy. In Section 4, we investigate the radial motion of colliding particles with the critical angular momentum in detail. The extremal and nonextremal cases are examined, respectively. The conclusion is given in the last section.

\section{The $2+1$ Dimensional BTZ Black Hole}

In this section we would like to study the horizon structure of the $2+1$ dimensional BTZ black hole. The metric of the BTZ black hole is usually written as [31] (with units $c=G=1$ )

$$
d s^{2}=-N_{r}^{2} d t^{2}+N_{r}^{-2} d r^{2}+r^{2}\left(N_{\phi} d t+d \phi\right)^{2}
$$

with

$$
\begin{aligned}
& N_{r}^{2}(r)=-M+\frac{r^{2}}{l^{2}}+\frac{J^{2}}{4 r^{2}}, \\
& N_{\phi}(r)=-\frac{J}{2 r^{2}},
\end{aligned}
$$

where $M$ and $J$ are the mass and spin angular momentum of the black hole, respectively, and $l^{2}$ is related to the cosmological constant $\Lambda$ by $l^{-2}=-\Lambda$.

The horizons can be solved from $\left.N_{r}\right|_{r=r_{h}}=0$, and they are given by

$$
r_{ \pm}=\sqrt{\frac{l}{2}\left(l M \pm \sqrt{l^{2} M^{2}-J^{2}}\right)} .
$$

Here, $r_{+}$is the outer horizon and $r_{-}$is the inner horizon. The existence of the horizon requires

$$
|J| \leq M l \text {. }
$$

The horizon of the extremal black hole (corresponding to $|J|=M l)$ is read as

$$
r_{e}=\sqrt{\frac{M}{2}} l
$$

\section{The Center-of-Mass Energy for the On-Horizon Collision}

To investigate the CM energy of the collision on the horizon of the BTZ black hole, we have to derive the $2+1$ dimensional "4-velocity" component of the colliding particle in the background of the $2+1$ dimensional BTZ black hole.

The generalized momentum $P_{\mu}$ is

$$
P_{\mu}=g_{\mu \nu} \dot{x}^{\nu}
$$

where the dot denotes the derivative with respect to the affine parameter $\lambda$ and $\mu, v=t, r, \phi$. Thus, the components $P_{t}$ and $P_{\phi}$ of the momentum are turned out to be

$$
\begin{gathered}
P_{t}=g_{t t} \dot{t}+g_{t \phi} \dot{\phi}, \\
P_{\phi}=g_{\phi \phi} \dot{\phi}+g_{t \phi} \dot{t} .
\end{gathered}
$$

$P_{t}$ and $P_{\phi}$ are constants of motion. In fact, they correspond to the test particle's energy per unit mass $E$ and the angular momentum parallel to the symmetry axis per unit mass $L$, respectively. And in the following discussion we will just regard these two constants of motion as $-E \equiv P_{t}$ and $L \equiv P_{\phi}$ [24].

The affine parameter $\lambda$ can be related to the proper time by $\tau=\mu \lambda$, where $\tau$ is given by the normalization condition $-\mu^{2}=g_{\mu \nu} \dot{x}^{\mu} \dot{x}^{\nu}$ with $\mu^{2}=1$ for time-like geodesics and $\mu^{2}=0$ for null geodesics. For a time-like geodesic, the affine parameter can be identified with the proper time, and thus, from (7), we can solve the $2+1$ dimensional "4-velocity" components $\dot{t}$ and $\dot{\phi}$ (where the dot denotes a derivative with respect to the proper time now) as

$$
\begin{aligned}
& \frac{d t}{d \tau}=\frac{2 E-\left(J L / r^{2}\right)}{2 N_{r}^{2}}, \\
& \frac{d \phi}{d \tau}=\frac{J\left(-J L+2 E r^{2}\right)+4 L r^{2} N_{r}^{2}}{4 r^{4} N_{r}^{2}} .
\end{aligned}
$$

For the remaining component $\dot{r}=d r / d \tau$ of the radial motion, we can obtain it from the Hamilton-Jacobi equation of the time-like geodesic:

$$
\frac{\partial S}{\partial \tau}=-\frac{1}{2} g^{\mu \nu} \frac{\partial S}{\partial x^{\mu}} \frac{\partial S}{\partial x^{\nu}}
$$

with the ansatz

$$
S=\frac{1}{2} \tau-E t+L \phi+S_{r}(r)
$$

where $S_{r}(r)$ is a function of $r$. Inserting the ansatz into (9), with the help of the metric (1), we get

$$
\left(\frac{d S_{r}(r)}{d r}\right)^{2}=\frac{J^{2} L^{2}-4 E J L r^{2}-4 r^{2}\left[L^{2} N_{r}^{2}+\left(-E^{2}+N_{r}^{2}\right) r^{2}\right]}{4 N_{r}^{4} r^{4}} .
$$

On the other hand, we have

$$
\frac{d S_{r}(r)}{d r}=P_{r}=g_{r r} \dot{r}=\frac{\dot{r}}{N_{r}^{2}} .
$$

Thus we get the square of the 4-velocity radial component:

$$
\left(\frac{d r}{d \tau}\right)^{2}=\frac{K^{2}-4 r^{2} N_{r}^{2}\left(L^{2}+r^{2}\right)}{4 r^{4}}
$$

where

$$
K=J L-2 E r^{2}
$$

Here we have obtained all nonzero $2+1$ dimensional " 4 velocity" components for the geodesic equation. Next we would like to study the CM energy of the two-particle collision in the background of the BTZ black hole. Here we consider a more general case that the two colliding particles have different energies $E_{1}$ and $E_{2}$ and different 
angular momenta per unit mass $L_{1}$ and $L_{2}$. For simplicity, the particles under consideration have the same rest mass $m_{0}$. We can compute the $\mathrm{CM}$ energy $E_{\mathrm{CM}}$ of this two-particle collision by using

$$
E_{\mathrm{CM}}=\sqrt{2} m_{0} \sqrt{1-g_{\mu \nu} u_{1}^{\mu} u_{2}^{\nu}}
$$

where $u_{1}^{\mu}$ and $u_{2}^{v}$ are the "4-velocity" vectors of the two particles $(u=(\dot{t}, \dot{r}, \dot{\phi}))$. With the help of (8) and (13), we obtain the CM energy:

$$
\begin{aligned}
\frac{E_{\mathrm{CM}}^{2}}{2 m_{0}^{2}}=\frac{1}{4 r^{4} N_{r}^{2}}[ & \left(J L_{1}-2 E_{1} r^{2}\right)\left(J L_{2}-2 E_{2} r^{2}\right) \\
& \left.+4 r^{2} N_{r}^{2}\left(-L_{1} L_{2}+r^{2}\right)-H_{1} H_{2}\right],
\end{aligned}
$$

where

$$
\begin{array}{r}
H_{i}=\sqrt{\left(J L_{i}-2 E_{i} r^{2}\right)^{2}-4 r^{2} N_{r}^{2}\left(L_{i}^{2}+r^{2}\right)} \\
(i=1,2) .
\end{array}
$$

For simplicity, we can rescale the $\mathrm{CM}$ energy as $\bar{E}_{\mathrm{CM}}^{2} \equiv$ $\left(1 / 2 m_{0}^{2}\right) E_{\mathrm{CM}}^{2}$. We would like to study $\bar{E}_{\mathrm{CM}}^{2}$ for the case that the particles collide on the black hole's horizon, which means $N_{r}=0$. The denominator of the expression on the right hand of (16) is zero, and the numerator of it is

$$
\begin{gathered}
K_{1} K_{2}-\sqrt{K_{1}^{2}} \sqrt{K_{2}^{2}}, \\
K_{i}=\left.K\right|_{E=E_{i}, L=L_{i}}, \quad i=1,2 .
\end{gathered}
$$

When $K_{1} K_{2} \geq 0$, the numerator will be zero and the value of $\bar{E}_{\mathrm{CM}}^{2}$ on the horizon will be undetermined, but when $K_{1} K_{2}<$ 0 , the numerator will be a negative finite value and $\bar{E}_{\mathrm{CM}}^{2}$ on the horizon will be negative infinity. So it should have $K_{1} K_{2} \geq 0$, and, for the CM energy on the horizon, we have to compute the limiting value of (16) as $r \rightarrow r_{h}$, where $r_{h}$ is the horizon of the black hole.

After some calculations, we get the limiting value of (16):

$$
\begin{aligned}
& \bar{E}_{\mathrm{CM}}^{2}\left(r \longrightarrow r_{h}\right) \\
& =2+\frac{\left(L_{1}-L_{2}\right)^{2}-l^{2}\left(E_{1}-E_{2}\right)^{2}-2\left(L_{1}-L_{2}\right)\left(L_{C 1}-L_{C 2}\right)}{2\left(L_{1}-L_{C 1}\right)\left(L_{2}-L_{C 2}\right)} \\
& \quad+\frac{l\left[\left(E_{2} L_{1}-E_{1} L_{2}\right)^{2}+M l^{2}\left(E_{1}-E_{2}\right)^{2}\right]\left(l M+\sqrt{l^{2} M^{2}-J^{2}}\right)}{J^{2}\left(L_{1}-L_{C 1}\right)\left(L_{2}-L_{C 2}\right)},
\end{aligned}
$$

which can also be rewritten as

$$
\bar{E}_{\mathrm{CM}}^{2}\left(r \longrightarrow r_{h}\right)=2+\frac{A}{2 K_{1} K_{2}},
$$

where

$$
\begin{aligned}
A= & J^{2}\left[\left(L_{1}-L_{2}\right)^{2}-\left(E_{1}-E_{2}\right)^{2} l^{2}\right. \\
& \left.-2\left(L_{1}-L_{2}\right)\left(L_{C 1}-L_{C 2}\right)\right] \\
& +2 l\left[\left(E_{2} L_{1}-E_{1} L_{2}\right)^{2}+\left(E_{1}-E_{2}\right)^{2} l^{2} M\right] \\
& \times\left(l M+\sqrt{l^{2} M^{2}-J^{2}}\right) .
\end{aligned}
$$

So it can be seen that when $K_{i}=0$, the CM energy on the horizon will blow up. Solving $K_{i}=0$, we get the critical angular momentum:

$$
L_{C i}=\frac{2 r_{h}^{2} E_{i}}{J}=\frac{E_{i} l\left(l M+\sqrt{l^{2} M^{2}-J^{2}}\right)}{J}, \quad i=1,2 .
$$

It is easy to prove that when $K_{1}=0$ and $K_{2}=0$, the CM energy is finite. So in order to obtain an arbitrarily high CM energy, one and only one of the colliding particles should have the critical angular momentum. For the extremal BTZ black hole $J=l M$, the $\bar{E}_{\mathrm{CM}}^{2}$ on the extremal horizon is

$$
\begin{aligned}
& \bar{E}_{\mathrm{CM}}^{2}\left(r \longrightarrow r_{e}\right) \\
& =2+\frac{M\left[\left(L_{1}-E_{1} l\right)-\left(L_{2}-E_{2} l\right)\right]^{2}+2\left(E_{2} L_{1}-E_{1} L_{2}\right)^{2}}{2 M\left(L_{1}-E_{1} l\right)\left(L_{2}-E_{2} l\right)} .
\end{aligned}
$$

Obviously, when one particle has the critical angular momentum $L_{\mathrm{C} 1}=E_{1} l$ (or $L_{\mathrm{C} 2}=E_{2} l$ ) and the other does not, the CM energy on the extremal horizon could be infinite.

From the above derivation, it seems that the CM energy could blow up on the horizon. However, in order to get arbitrarily high CM energy on the horizon of the BTZ black hole, the colliding particle with the critical angular momentum must be able to reach the horizon of the black hole. We will investigate this part in the next section.

\section{The Radial Motion of the Particle with the Critical Angular Momentum near the Horizon}

In this section, we will study the radial motion of the particle with the critical angular momentum and find the region where it can exist. In order for a particle to reach the horizon of the black hole, the square of the radial component of the "4-velocity" $(d r / d \tau)^{2}$ in (13) has to be positive in the neighborhood of the black hole's horizon. Obviously, $\left.R(r)\right|_{L=L_{C i}}=0$ on the horizon of the BTZ black hole. For a particle with arbitrary energy $E$ and angular momentum $L$, the explicit form of $(d r / d \tau)^{2}$, which is denoted by $R(r)$, reads

$$
\begin{aligned}
R(r) \equiv & \left(\frac{d r}{d \tau}\right)^{2}=E^{2}-\frac{L^{2}}{l^{2}}+M \\
& +\frac{1}{r^{2}}\left(L^{2} M-E J L-\frac{J^{2}}{4}\right)-\frac{r^{2}}{l^{2}} .
\end{aligned}
$$




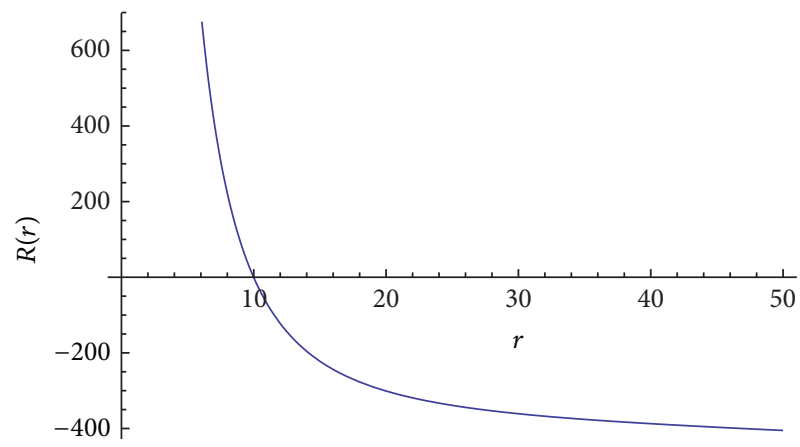

(a)

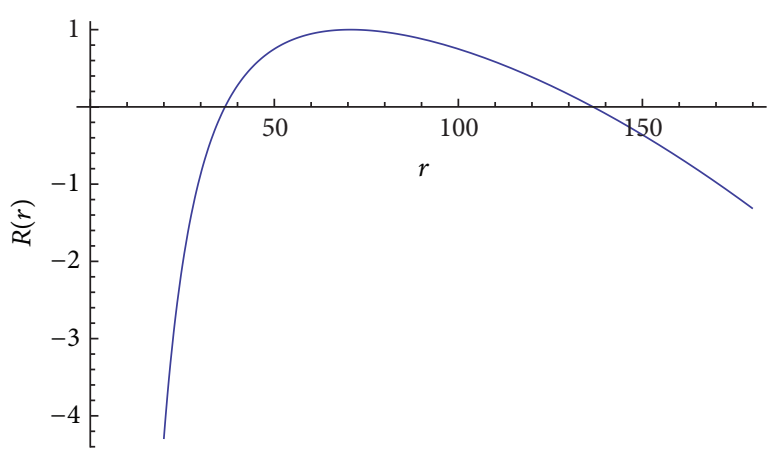

(b)

FIgURE 1: Behaviors of $R(r)$ for (a) $L^{2} M-E J L-\left(J^{2} / 4\right)>0$ and (b) $L^{2} M-E J L-\left(J^{2} / 4\right)<0$.

We draw $R(r)$ in Figure 1. It can be seen that, when $L^{2} M-$ $E J L-J^{2} / 4>0, R(r \rightarrow 0) \rightarrow+\infty$ and $R(r \rightarrow+\infty) \rightarrow-\infty$, so there is only one positive root for $R(r)=0$ and the particle can exist in the region inside of the root. When $L^{2} M-E J L-$ $J^{2} / 4<0, R(r \rightarrow 0) \rightarrow-\infty$ and $R(r \rightarrow+\infty) \rightarrow-\infty$, and there are two positive roots and the particle can exist in the region between the two roots. The bigger root of $R(r)=0$ is

$$
\begin{aligned}
r_{2}= & \left(l^{2}\left(E^{2}+M\right)-L^{2}\right. \\
& \left.+\sqrt{\left[l^{2} M-J l+(L-E l)^{2}\right]\left[l^{2} M+J l+(L+E l)^{2}\right]}\right)^{1 / 2} \\
& \times(\sqrt{2})^{-1} .
\end{aligned}
$$

We find that it increases with $E$ and $L$, which means that the particle can move arbitrarily far from black hole's horizon with its energy and angular momentum's increase.

Next, we will study the radial motion of the particle with the critical angular momentum:

$$
\begin{aligned}
\left.R(r)\right|_{L=L_{c}}= & \frac{W}{r^{2}}-\frac{r^{2}}{l^{2}}+2 E^{2}+M \\
& -\frac{2 E^{2} l^{2} M^{2}+2 E^{2} l M \sqrt{l^{2} M^{2}-J^{2}}}{J^{2}},
\end{aligned}
$$

where

$$
\begin{aligned}
W= & \frac{E^{2} l\left[2 l M\left(l^{2} M^{2}-J^{2}\right)+\left(2 l^{2} M^{2}-J^{2}\right) \sqrt{l^{2} M^{2}-J^{2}}\right]}{J^{2}} \\
& -\frac{J^{2}}{4} .
\end{aligned}
$$

By solving $W=0$ we get the critical energy $E_{0}$ :

$$
E_{0}=\frac{J^{2}}{2 \sqrt{2 l^{2} M\left(l^{2} M^{2}-J^{2}\right)+\left(2 l^{3} M^{2}-J^{2} l\right) \sqrt{l^{2} M^{2}-J^{2}}}} .
$$

When $E>E_{0}, R(r)=0$ has one root

$$
\begin{aligned}
r_{0}=\frac{1}{\sqrt{2} J}\left\{l ^ { 2 } \left[J^{2} M\right.\right. & +2 E^{2} \\
& \left.\times\left(J^{2}-l M\left(l M+\sqrt{l^{2} M^{2}-J^{2}}\right)\right)\right] \\
+ & l\left(l^{2} M^{2}-J^{2}\right) \\
\times & {\left[J^{4}+8 E^{4} l^{3} M\left(l M+\sqrt{l^{2} M^{2}-J^{2}}\right)\right.} \\
+4 E^{2} J^{2} l\left(l M-E^{2} l\right. & \left.\left.\left.\left.+\sqrt{l^{2} M^{2}-J^{2}}\right)\right]\right]^{1 / 2}\right\}^{1 / 2}
\end{aligned}
$$

and the particle with the critical angular momentum can exist inside of it. When $E<E_{0}, R(r)=0$ has two roots

$$
r_{0+}=\frac{1}{\sqrt{2} J}\left\{l ^ { 2 } \left[J^{2} M+2 E^{2}\right.\right.
$$

$$
\begin{array}{r}
\left.\times\left(J^{2}-l M\left(l M+\sqrt{+l^{2} M^{2}-J^{2}}\right)\right)\right] \\
+l\left[\left(l^{2} M^{2}-J^{2}\right)\right. \\
\times\left(J^{4}+8 E^{4} l^{3} M\left(l M+\sqrt{l^{2} M^{2}-J^{2}}\right)\right. \\
+4 E^{2} J^{2} l\left(l M-E^{2} l\right. \\
\left.\left.\left.\left.+\sqrt{l^{2} M^{2}-J^{2}}\right)\right)\right]^{1 / 2}\right\}^{1 / 2},
\end{array}
$$




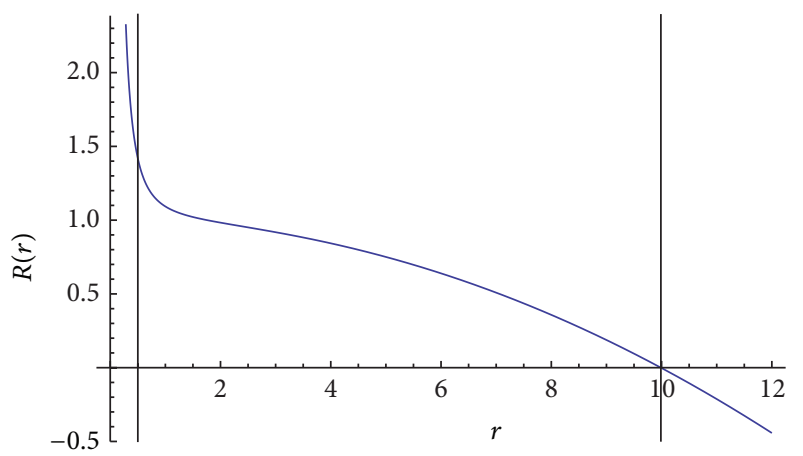

(a)

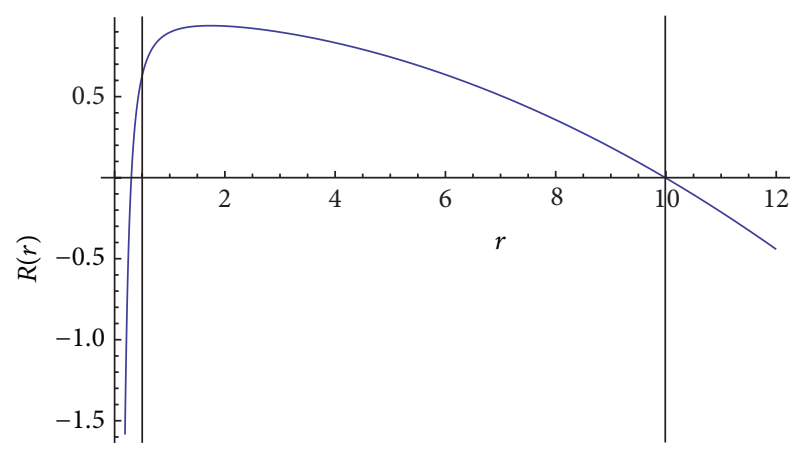

(b)

FIGURE 2: The variation of $R(r)$ versus radius $r$ for the case of the nonextremal BTZ black hole $(l=10, M=1$, and $J=1), E>E_{0}(($ a) $E=0.003)$ and $E<E_{0}((\mathrm{~b}) E=0.002)$. The vertical lines denote the locations of the outer and inner horizons.

$$
\begin{aligned}
& r_{0-}=\frac{1}{\sqrt{2} J}\left\{l ^ { 2 } \left[J^{2} M+2 E^{2}\right.\right. \\
& \left.\times\left(J^{2}-l M\left(l M+\sqrt{+l^{2} M^{2}-J^{2}}\right)\right)\right] \\
& -l\left[\left(l^{2} M^{2}-J^{2}\right)\right. \\
& \times\left(J^{4}+8 E^{4} l^{3} M\left(l M+\sqrt{l^{2} M^{2}-J^{2}}\right)\right. \\
& +4 E^{2} J^{2} l\left(l M-E^{2} l\right. \\
& \left.\left.\left.\left.+\sqrt{l^{2} M^{2}-J^{2}}\right)\right)\right]^{1 / 2}\right\}^{1 / 2},
\end{aligned}
$$

and the particle with the critical angular momentum can exist between them. The above discussion only concerns the square of the "4-velocity" radial component. To find whether the particle with the critical angular momentum can reach the horizon of the BTZ black hole, we should investigate the roots of $R(r)=0$ and the horizons of the black hole. The nonextremal and extremal cases will be considered in the following.

4.1. Nonextremal BTZ Black Hole. For the nonextremal BTZ black hole case, we can prove that the solution (for $E>E_{0}$ case) or the bigger solution (for $E<E_{0}$ case) of $R(r)=0$ is just the outer horizon of black hole:

$$
r_{0}=r_{+}=\sqrt{\frac{l}{2}\left(l M+\sqrt{l^{2} M^{2}-J^{2}}\right)} .
$$

That means that the particle with the critical angular momentum can exist inside the outer horizon of the nonextremal BTZ black hole.

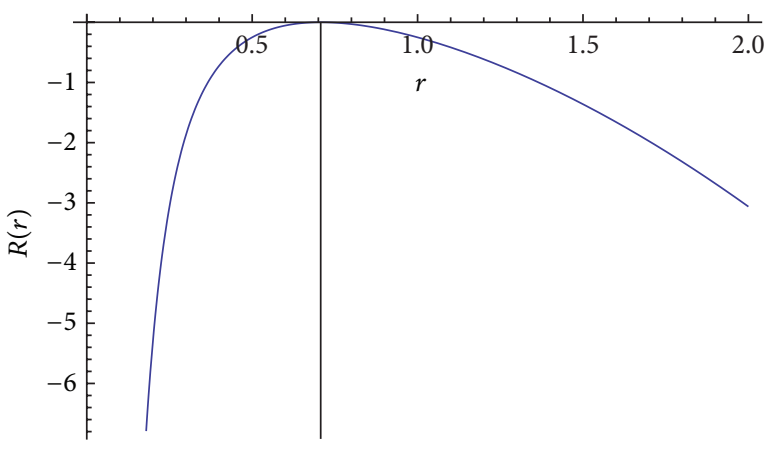

FIGURE 3: The variation of $R(r)$ versus radius $r$ for the case of the extremal BTZ black hole $(l=1, M=1$, and $J=1)$. The vertical line denotes the locations of the degenerated horizon.

4.2. Extremal BTZ Black Hole. For the extremal BTZ black hole case, $R(r)$ for particle with the critical angular momentum becomes very simple:

$$
R(r)=M-\frac{r^{2}}{l^{2}}-\frac{J^{2}}{4 r^{2}}
$$

We solve $R(r)=0$ and get

$$
r_{0}=\sqrt{\frac{M}{2}} l \text {. }
$$

It is just the degenerated horizon of the extremal black hole.

The behaviors of $R(r)$ for the particle with the critical angular momentum are plotted in Figure 2 for the nonextremal black hole and Figure 3 for the extremal black hole. For the nonextremal black hole, we find that the particle with the critical angular momentum can exist inside the outer horizon. So particle collision on the inner horizon could produce unlimited CM energy. For the extremal black hole, the particle with the critical angular momentum could only exist on the degenerated horizon, so if such particle exists, then unlimited CM energy will be approached. 


\section{Conclusion}

In this work, we have analyzed the possibility that the $2+1$ dimensional BTZ black holes can serve as particle accelerator. We first calculate the CM energy for the two-particle collision. In order to obtain unlimited CM energy, one of the particles should have the critical angular momentum. Next, we study the radial motion for the particle with the critical angular momentum. For the extremal BTZ black hole, particles with critical angular momentum can only exist on the outer horizon of the BTZ black hole. So if such particle exists, then unlimited CM energy will be approached. For the nonextremal BTZ black hole, particles can collide on the inner horizon with arbitrarily high CM energy.

\section{Conflict of Interests}

The authors declare that there is no conflict of interests regarding the publication of this paper.

\section{Acknowledgments}

This work was supported by the National Natural Science Foundation of China (Grants no. 11205074 and no. 11375075) and the Fundamental Research Funds for the Central Universities (Grants no. lzujbky-2013-18 and no. lzujbky-2013-21).

\section{References}

[1] M. Banãdos, J. Silk, and S. M. West, "Kerr black holes as particle accelerators to arbitrarily high energy," Physical Review Letters, vol. 103, Article ID 111102, 2009.

[2] E. Berti, V. Cardoso, L. Gualtieri, F. Pretorius, and U. Sperhake, "Comment on :kerr black holes as particle accelerators to arbitrarily high energy," Physical Review Letters, vol. 103, no. 23, Article ID 239001, 2009.

[3] T. Jacobson and T. P. Sotiriou, "Spinning black holes as particle accelerators," Physical Review Letters, vol. 104, no. 2, Article ID 021101, 2010.

[4] A. A. Grib and Y. V. Pavlov, "On particlecollisions in the gravitational field of the Kerr black hole," Astroparticle Physics, vol. 34, no. 7, pp. 581-586, 2011.

[5] K. Lake, "Particle Accelerators inside Spinning Black Holes," Physical Review Letters, vol. 104, Article ID 211102, 2010.

[6] K. Lake, "Erratum to particle accelerators inside spinning black holes," Physical Review Letters, vol. 104, Article ID 259903, 2010.

[7] A. A. Grib and Y. V. Pavlov, "On the collisions between particles in the vicinity of rotating black holes," JETP Letters, vol. 92, no. 3, pp. 125-129, 2010.

[8] S. W. Wei, Y. X. Liu, H. Guo, and C. E. Fu, "Charged spinning black holes as particle accelerators," Physical Review D, vol. 82, Article ID 103005, 2010.

[9] A. A. Grib and V. Yu. Pavlov, "On particle collisions nearKerr's black holes," http://arxiv.org/abs/1007.3222 .

[10] O. B. Zaslavskii, "Acceleration of particles by nonrotating charged black holes," JETP Letters, vol. 92, no. 9, pp. 571-574, 2011.
[11] S. W. Wei, Y. X. Liu, H. T. Li, and F. W. Chen, "Particle collisions on stringy black hole background," Journal of High Energy Physics, vol. 12, article 066, 2010.

[12] P. J. Mao, L. Y. Jia, J. R. Ren, and R. Li, "Acceleration of particles in Einstein-Maxwell-Dilaton black hole," http://arxiv.org/abs/1008.2660 .

[13] T. Harada and M. Kimura, "Collision of an innermost stable circular orbit particle around a Kerr black hole," Physical Review D, vol. 83, no. 2, Article ID 024002, 2011.

[14] T. Harada and M. Kimura, "Collision of two general geodesic particles around a Kerr black hole," Physical Review D, vol. 83, no. 8, Article ID 084041, 2011.

[15] A. A. Grib and Y. V. Pavlov, "On particle collisions near rotating black holes," Gravitation and Cosmology, vol. 17, no. 1, pp. 42-46, 2011.

[16] M. Bañados, B. Hassanain, J. Silk, and S. M. West, "Emergent flux from particle collisions near a Kerr black hole," Physical Review D, vol. 83, no. 2, Article ID 023004, 2011.

[17] M. Patil and P. S. Joshi, "Naked singularities as particle accelerators," Physical Review D, vol. 82, Article ID 104049, 2010.

[18] M. Patil, P. S. Joshi, and D. Malafarina, "Naked singularities as particle accelerators. II," Physical Review D, vol. 83, Article ID 064007, 2011.

[19] O. B. Zaslavskii, "Acceleration of particles as a universal property of rotating black holes," Physical Review D, vol. 82, no. 8, Article ID 083004, 2010.

[20] O. B. Zaslavskii, "Acceleration of particles by black holes: a general explantion," Classical and Quantum Gravity, vol. 28, no. 10, Article ID 105010, 2011.

[21] Y. Zhu, S.-F. Wu, Y.-X. Liu, and Y. Jiang, "General stationary charged black holes as charged particle accelerators," Physical Review D, vol. 84, no. 4, Article ID 043006, 2011.

[22] Y. Zhu, S.-F. Wu, Y. Jiang, and G.-H. Yang, "Can the ReissnerNordström black hole or Schwarzschild black hole be the stable Planck-scale particle accelerator?” Physical Review D, vol. 84, no. 12, Article ID 123002, 2011.

[23] M. Kimura, K.-I. Nakao, and H. Tagoshi, "Acceleration of colliding shells around a black hole: validity of the test particle approximation in the Banados-Silk-West process," Physical Review D, vol. 83, no. 4, Article ID 044013, 2011.

[24] E. Hackmann, C. Lämmerzahl, V. Kagramanova, and J. Kunz, "Analytical solution of the geodesic equation in Kerr-(anti-) de Sitter space-times," Physical Review D, vol. 81, no. 4, Article ID 044020, 2010.

[25] S. Fernando, "String black hole: can it be a particle accelerator?" General Relativity and Gravitation, vol. 46, no. 1, Article ID 1634, 2014.

[26] M. Sharif and N. Haider, "Study of center of mass energy by particles collision in some black holes," Astrophysics and Space Science, vol. 346, pp. 111-117, 2013.

[27] Z. Stuchlík and J. Schee, "Ultra-high-energy collisions in the superspinning Kerr geometry," Classical and Quantum Gravity, vol. 30, no. 7, Article ID 075012, 2013.

[28] C. Ding, C. Liu, and Q. Quo, "Spacetime noncommutative effect on black hole as particle accelerators," International Journal of Modern Physics D, vol. 22, no. 4, Article ID 1350013, 2013.

[29] M. Bejger, T. Piran, M. Abramowicz, and F. Hakanson, "Collisional Penrose process near the horizon of extreme Kerr black holes," Physical Review Letters, vol. 109, Article ID 121101, 2012. 
[30] Y. Li, J. Yang, Y.-L. Li, S.-W. Wei, and Y.-X. Liu, "Particle acceleration in Kerr-(anti-)de Sitter black hole backgrounds," Classical and Quantum Gravity, vol. 28, no. 22, Article ID 225006, 2011.

[31] M. Bañados, C. Teitelboim, and J. Zanelli, "Black hole in threedimensional spacetime," Physical Review Letters, vol. 69, no. 13, pp. 1849-1851, 1992. 

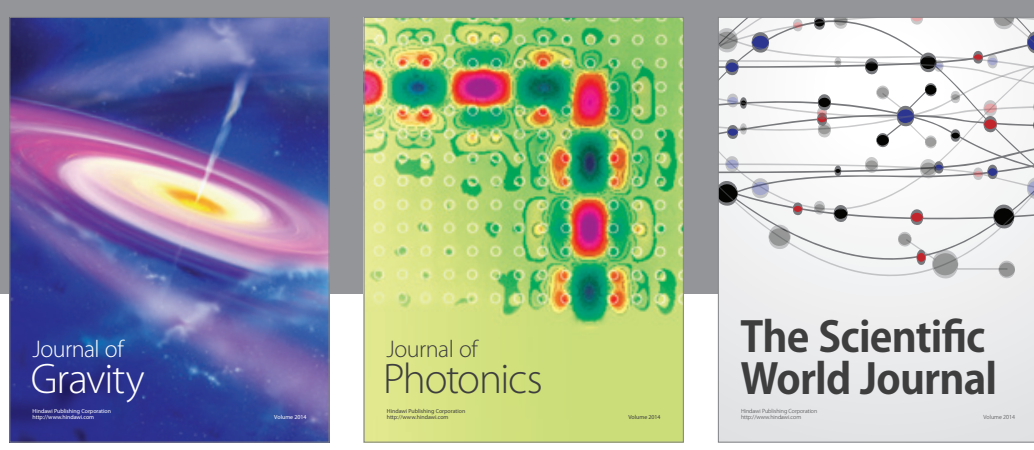

The Scientific World Journal
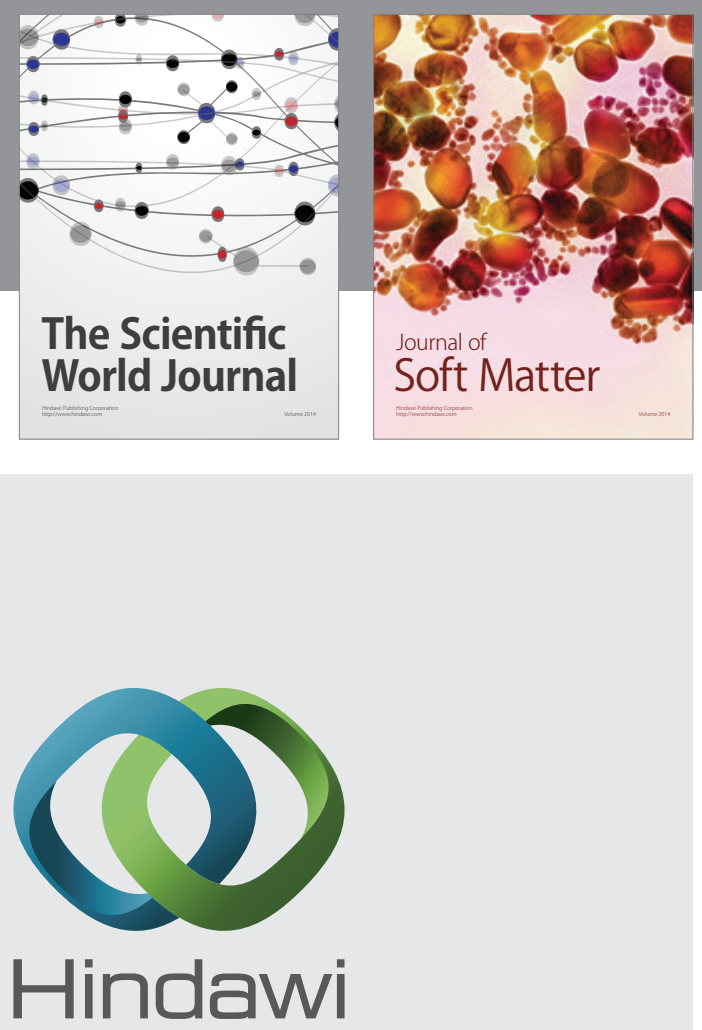

Submit your manuscripts at

http://www.hindawi.com

nternational Journal of

Statistical Mechanics
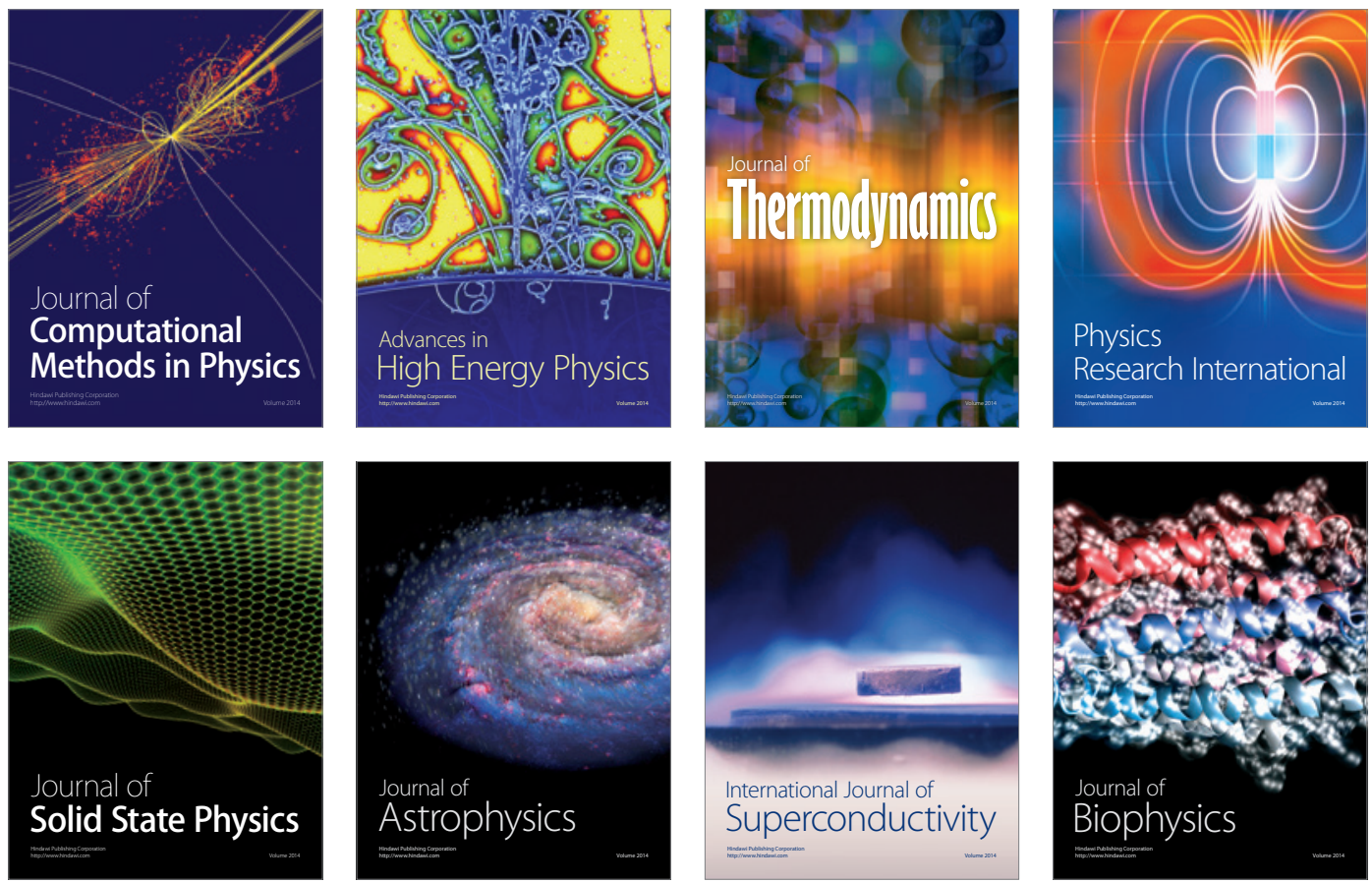
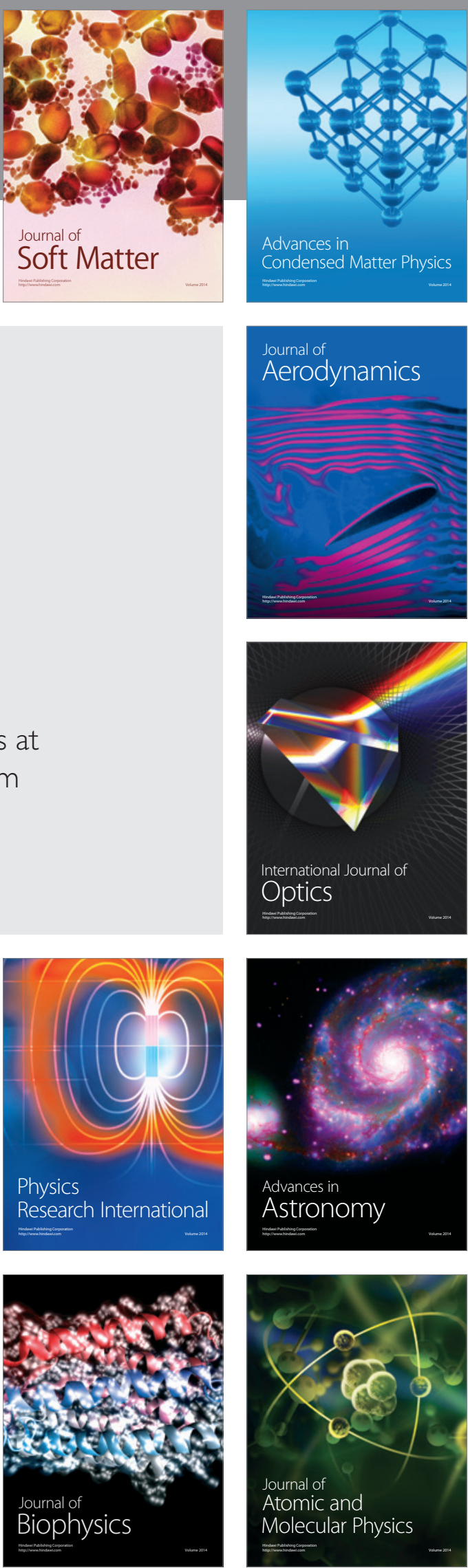